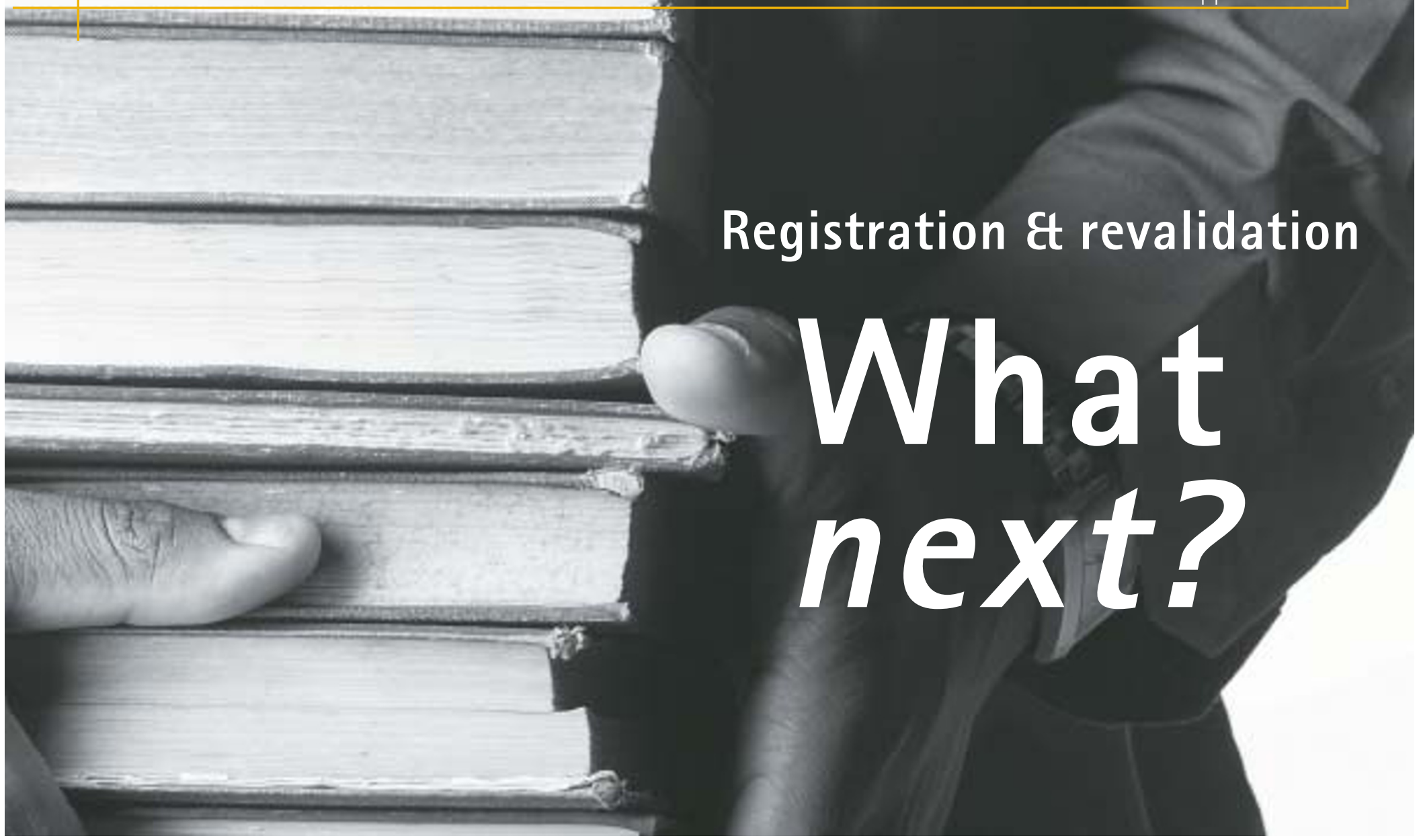

8 ULRIKE

MATTHESIUS

looks at the

changes

awaiting

dentists with

regard to

registration

and

revalidation
$\mathrm{S}$ ince the late 1990s, there has been a renewed focus on the need for change in the way in which healthcare professionals are assessed for their fitness to practise. Mandatory CPD requirements, such as the GDC's new Lifelong Learning Scheme, are already no longer enough. The final report of the Bristol Inquiry into children's heart surgery, recommended that:

'Periodic revalidation, whereby healthcare professionals demonstrate that they remain fit to practise in their chosen profession, should be compulsory for all healthcare professionals. The requirement to participate in periodic revalidation should be included in the contract of employment.

In its response, the Government made a commitment to work with the professional regulators on appropriate systems of revalidation.

The first changes were introduced with the advent of the General Dental Council's 'Lifelong Learning Scheme' and its requirement for mandatory continuing professional development.

The GDC has now started work on developing a revalidation scheme for dentists. It is in its very early stages, and further consultations will follow.

\section{Why revalidation?}

Traditionally, registration has been based on the assumption that a qualified professional is fit to practise indefinitely, unless evidence to the contrary is received. At the point of initial registration, it is accepted that the criteria are stringent enough to guarantee a professional's high standard of training and ability, but until recently there was no need for a dentist to take any postgraduate training if they did not wish to do so.

This system of 'registration for life unless adverse proof is received' was designed in a time when the judgement of health professionals was seldom challenged. But times have changed. A more inquisitive public and the development of new treatments and technology have motivated the regulators to think about new approaches to regulation.

The GDC has already begun to take action in this area, with the introduction of mandatory CPD, the reforms to fitness to practise procedures, and the development of procedures to remedy problems of poor performance.

More needs to be done. It is nowadays necessary to show that a professional remains fit to practise, that is there is a need for quality assurance of those who remain on the register. Mandatory CPD was a step in this direction, but a revalidation scheme will be the next development designed to ensure that dentists are up-to-date with the latest scientific developments and procedures.

As well as accepting that changes need to be made, the GDC recognises the need not to make any new procedures too elaborate and thus take away time from the dentist's main objective - the treatment of patients. It has therefore committed itself to making any new procedures as simple as possible. The aim is not to set a 'gold standard' for all dentists, but to carry out revalidation at an appropriate and reasonable level.

\section{Draft principles}

Any scheme would tie in very closely with the current procedures already in place at the GDC: the 
Lifelong Learning Scheme and the fitness to practise procedures.

While no decision has been made yet as to the formalities of any scheme, some general principles have been outlined, firmly focussing on fitness to practise. It will not be the role of the scheme to set new or unrealistic standards. Assurance of competence in current performance will be sought during the process. It will tie in with local quality assurance mechanisms, for example, assessment systems of postgraduate deans and employers' appraisal systems. The system will also need to have backing both from dentists and PCDs, public and private sector employers, the Government and consumer organisations. Wherever possible, the GDC system will also try to be consistent with systems of other health regulators.

\section{The GMC model}

The GMC has now embarked on the process of revalidation for all doctors. The formal system will be up and running from 1 January 2005 and will focus on a licence to practise based on periodic revalidation. It will look at the way in which a doctor's day-today practice reflects the principles outlined in Good Medical Practice. Revalidation is set to take place every five years.

The GMC model foresees two different routes for doctors: the appraisal route or the independent route.

\section{Appraisal route}

The majority of doctors are expected to take the appraisal route. Doctors taking this route need to work in a managed environment and participate in annual appraisal, based on Good Medical Practice and in a quality assurance framework. Documentation must be provided to the GMC. For those who take this route, no further collection of evidence is necessary beyond that for the annual appraisal.

\section{Independent route}

Doctors must demonstrate their adoption of the principles of Good Medical Practice by providing paperwork to prove their fitness to practise and their undertaking of appropriate CPD.

\section{Registration}

Doctors who do not wish to have a licence to practise can stay on the register, but will not be able to practice. They still have to adhere to professional obligations and ethics.

\section{Results of revalidation}

The possibilities are:

- Licence will remain valid or

- Additional information is needed before it can be renewed or

- The information provided is not persuasive; assessment of fitness to practise is necessary. If there is evidence of a lack of fitness to practise,

\section{'A more inquisitive public and the \\ the relevant procedure will be called in, or \\ - Withdrawal of licence: if it is no longer wanted, if the appropriate fee is not paid, if the doctor fails to take part in revalidation when asked, or if a fitness to practise panel has ruled that regis- tration should be suspended. There will be an appeals procedure.} development of new treatments and technology have motivated the regulators to think about new approaches to regulation.'
The GMC model may not be appropriate to dentistry. For example, there is at present no appraisal scheme for GDPs (unlike medical GPs). Unless such a scheme were to be introduced, GDPs would be required to use the independent route which would be less straightforward, and this is undesirable for a dentistry model. Despite this, it might be possible to use some aspects of the scheme for the purposes of revalidation of dentists, and the GDC is looking at this and other professions' models to draw upon.

A new consultation forum of all stakeholders is expected to be established at the end of 2003. A timescale for a new scheme has not been set, but it is not expected to come into force until very late in the current decade. The main thing to remember is that the GDC is committed to keeping its system simple and to balance the protection of the public with a wish not to over-regulate the profession.

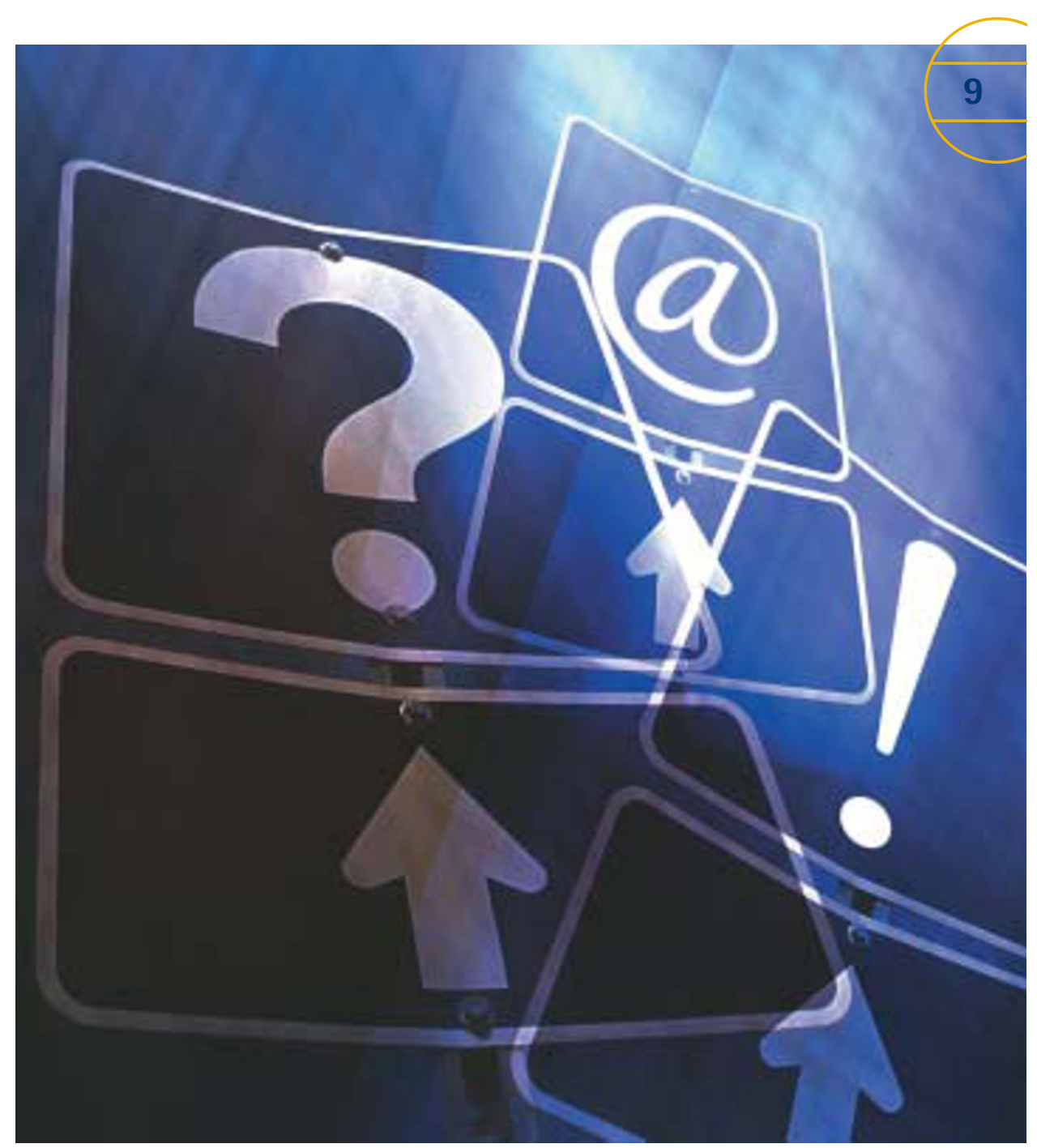

